\title{
Compreensão das características clínicas do COVID-19: uma revisão narrativa
}

\author{
Understanding the clinical characteristics of COVID-19: a narrative review
}

Comprender las características clínicas de COVID-19: una revisión narrativa

Suzana Bastos Jácome de Souza', Gustavo Medeiros Frota², Isabela Bastos Jácome de Souza², Aline Sharlon Maciel Batista Ramos ${ }^{3}$, Rafael Pavão Gonçalves ${ }^{4}$, Alba Angélica Nunes Mouta ${ }^{5 *}$, Virginia Araújo Albuquerque ${ }^{1}$, Augusto César Beltrão da Silva ${ }^{1}$, Priscila Favoritto Lopes ${ }^{5}$, Renata Paula Lima Beltrão $0^{1,5}$.

\section{RESUMO}

Objetivo: Conhecer através de uma revisão as características clínicas da COVID-19, sua relação e risco de gravidade de pacientes com doenças subjacentes. Revisão Bibliográfica: A epidemia que surgiu no final de 2019 na China tem apresentado padrões de distribuição que se repetem pelo mundo. Sua apresentação de maior gravidade tem se concentrado em extremos de idade, em especial nos mais velhos. A prevalência no gênero masculino levanta explicações genéticas e comportamentais. A infecção assintomática se mantém como desafio diagnóstico e elo é primordial na manutenção da epidemia. A apresentação clínica comumente engloba febre, tosse, dispneia, fadiga ou mialgia, e de forma menos frequentes os sintomas intestinais. Complicações como insuficiência respiratória, sepse e choque séptico possuem elevada mortalidade, especialmente em pacientes com comorbidades, como: hipertensão, o diabetes as doenças cardiovasculares, cerebrovasculares e doenças do aparelho respiratório. A Síndrome Respiratória Aguda Grave Coronavírus 2 (SARS-CoV-2) é desencadeada pela ligação da glicoproteína $S$ do vírus à Enzima Conversora de Angiotensina (ECA2), abundante no coração, nas células epiteliais do pulmão, intestino delgado, rins e vasos sanguíneos, justificando sua apresentação clínica. Considerações Finais: Admite-se o pouco conhecimento sobre o COVID-19, o que torna temerário o enfrentamento da grande pandemia, exigindo estudos complementares e mais assertivo.

Palavras-chave: Infecções por coronavírus, Pandemias, Sinais e sintomas.

\begin{abstract}
Objective: To know, through a review, the clinical characteristics of COVID-19, its relationship and risk of severity of patients with underlying diseases. Bibliographic Review: The epidemic that emerged in late 2019 in China has shown patterns of distribution that are repeated throughout the world. Its most serious presentation has been concentrated on extremes of age, especially in the elderly. The prevalence in males raises genetic and behavioral explanations. Asymptomatic infection remains a diagnostic challenge and the link is paramount in maintaining the epidemic. The clinical presentation commonly includes fever, cough,
\end{abstract}

\footnotetext{
${ }^{1}$ Instituto de Ensino Superior do Vale do Parnaíba (IESVAP). Parnaíba - PI.

¿Universidade Federal do Maranhão (UFMA). São Luís - MA.

3Universidade Estadual do Rio de Janeiro (UERJ). Rio de Janeiro - RJ.

${ }^{4}$ Centro Universitário do Maranhão (UNICEUMA). São Luís - MA.

5Universidade Federal do Delta do Parnaíba (UFDPar). Parnaíba - PI.

*E-mail: angelicanmouta@gmail.com
} 
dyspnoea, fatigue or myalgia, and intestinal symptoms are less frequent. Complications such as respiratory failure, sepsis and septic shock have high mortality, especially in patients with comorbidities, such as: hypertension, diabetes, cardiovascular and cerebrovascular diseases and diseases of the respiratory system. Severe Acute Respiratory Syndrome Coronavirus 2 (SARS-CoV-2) is triggered by the binding of the virus glycoprotein $S$ to the Angiotensin-Converting Enzyme (ACE2), abundant in the heart, in the epithelial cells of the lung, small intestine, kidneys and blood vessels, justifying its clinical presentation. Final Considerations: Little knowledge about COVID-19 is admitted, which makes the confrontation of the great pandemic reckless, requiring complementary and more assertive studies.

Keywords: Coronavirus infections, Pandemics, Signs and symptoms.

\section{RESUMEN}

Objetivo: Conocer, a través de una revisión, las características clínicas de COVID-19, su relación y el riesgo de gravedad de los pacientes con enfermedades subyacentes. Revisión bibliográfica: la epidemia que surgió a fines de 2019 en China ha mostrado patrones de distribución que se repiten en todo el mundo. Su presentación más grave se ha concentrado en los extremos de la edad, especialmente en los ancianos. La prevalencia en los hombres plantea explicaciones genéticas y de comportamiento. La infección asintomática sigue siendo un desafío diagnóstico y el vínculo es primordial para mantener la epidemia. La presentación clínica comúnmente incluye fiebre, tos, disnea, fatiga o mialgia, y los síntomas intestinales son menos frecuentes. Las complicaciones como la insuficiencia respiratoria, la sepsis y el shock séptico tienen una alta mortalidad, especialmente en pacientes con comorbilidades, tales como: hipertensión, diabetes, enfermedades cardiovasculares y cerebrovasculares y enfermedades del sistema respiratorio. Síndrome respiratorio agudo severo El coronavirus 2 (SARS-CoV-2) se desencadena por la unión de la glucoproteína S del virus a la enzima convertidora de angiotensina (ACE2), abundante en el corazón, en las células epiteliales del pulmón, intestino delgado, riñones y vasos sanguíneos. justificando su presentación clínica. Consideraciones finales: se admite poco conocimiento sobre COVID-19, lo que hace que la confrontación de la gran pandemia sea temeraria, requiriendo estudios complementarios y más asertivos.

Palabras-clave: Infecciones por coronavirus, Pandemias, Signos y síntomas.

\section{INTRODUÇÃO}

No final do mês dezembro de 2019, pessoas ligadas ao mercado de Frutos do Mar em Wuhan, na China, foram diagnosticadas com "pneumonias de origem desconhecida" (YANG J, et al., 2020; WU D, et al., 2020). Logo se descobriu que a origem do conjunto de casos de pneumonia era de etiologia viral e causada por um novo betacoronavírus recém-identificado, provisoriamente chamado 2019-nCoV e renomeado de Síndrome respiratória aguda grave - Coronavírus 2 (SARS-CoV2) pelo Grupo de Estudo para Coronavírus do Comitê Internacional de Taxonomia de Vírus, sendo a doença oficialmente nomeada pela Organização Mundial da Saúde (OMS) de doença do novo coronavírus 2019 (COVID-19) (GUO YR, et al., 2020; LAI CC, et al., 2020; WANG YXJ, et al., 2020).

Em janeiro de 2020, o SARS-CoV-2 se espalhou rapidamente na China, dentro e fora da província de Hubei e alcançou outros países, sendo declarada uma pandemia em 11 de março de 2020 pela OMS. O Relatório de situação global da COVID-2019 em 20 de abril de 2020 revela mais de 2.400 .000 mil casos confirmados de COVID-19, com um total de total 169.595 mortes em todo o mundo. Já acomete 185 países dentre os 193 existentes no mundo (NEHER RA, et al., 2020; HOPKINS J, 2020; WANG YXJ, et al., 2020). O surto da COVID-19 é considerado um desafio global por apresentar um rápido aumento de pacientes críticos, como ainda não existe um tratamento específico e devido ao alto poder de infectividade do novo vírus, os números de contaminados e de mortes cresce exponencialmente a cada dia, sendo potencialmente letal e impactando milhões de pessoa, tornando-se um grande problema de saúde (CUNNINGHAM AC, et al., 2020; VANKADARI N, WILCE JA, 2020). 
Milhares de pessoas estão sendo infectadas diariamente, o que acaba levando ao colapso dos sistemas de saúde de diversos países em todo o mundo. Os vírus do gênero $\beta$-coronavírus, do qual o SARS-CoV-2 faz parte, infectam mamíferos e são responsáveis por infecções respiratórias em humanos e enterites em animais (MALIK YS, et al., 2020). Os pacientes afetados pela COVID-19 apresentam sintomas similares aos da influenza, especialmente febre, sintomas respiratórios como tosse, falta de ar e ocasionalmente, diarreia aquosa, náuseas e vômitos (MALIK YS, et al., 2020; WANG YXJ, et al., 2020; YANG J, et al., 2020).

A transmissão se dá por gotículas respiratórias expelidas por meio da tosse e/ou espirro do paciente infectado com COVID-19 e por contato com fômites, ou seja, com objetos manipulados por pacientes infectados, que passam a conter o vírus e se tornam fonte de contaminação (YANG J, et al., 2020). Os levantamentos realizados por Fung SY, et al. (2020) e Wu D, et al. (2020) indicaram que o vírus pode se replicar no trato digestivo sugerindo a possibilidade de transmissão do SARS-CoV-2 pela via fecal-oral como ocorreu em 2003 nos casos da Síndrome respiratória aguda grave - coronavírus (SARS-CoV), porém ressaltam a necessidade de mais estudos para elucidar o papel exato da transmissão fecal-oral na disseminação da SARS-CoV-2.

Ainda não existe, até o momento, vacina ou tratamento antiviral específico eficaz para o tratamento de pacientes gravemente doentes, muitos estudos levantam o uso da associação de drogas como a cloroquina (ou seu derivado, a hidroxicloroquina) e a azitromicina, ainda existem estudos que são contra o uso desses medicamentos, mas muitos artigos compravam a eficácia dessa associação em tratamentos de pacientes infectados por COVID-19. Além dessas duas drogas, existem evidências mostrando que a associação com Sulfato de Zinco também pode ser benéfico para a melhoria do quadro dos pacientes; também existem evidências sobre o uso da Ivermectina e diversas outras drogas, no entanto, devido serem todos estudos preliminares que contam com uma população de estudo pequena, ainda existem muitas incertezas quanto ao tratamento. A prestação dos serviços de saúde consiste em cuidados de suporte, como a oxigenação, a ventilação mecânica e o gerenciamento de fluidos (CUNNINGHAM AC, et al., 2020; ESPER RN, et al., 2020; WANG YXJ, et al., 2020; WU D, et al., 2020).

A fim de aprofundar o conhecimento a respeito desta nova infecção causada pelo SARS-CoV-2, este estudo teve por objetivo conhecer as características clínicas da COVID-19, as características patogênicas do SARS-CoV2 e os aspectos relacionados a tratamento e prognóstico, avaliando o risco de gravidade de pacientes com doenças subjacentes e COVID-19.

\section{REVISÃO BIBLIOGRÁFICA}

\section{Patogenicidade da SARS-CoV-2}

Os coronavírus (CoVs) são encontrados em diferentes espécies de animais, dos quais os vírus 229E, OC43, HKU1, NL63, CoV da síndrome respiratória aguda grave (SARS) e CoV da síndrome respiratória do Oriente Médio (MERS) e mais recentemente o CoV da SARS-CoV-2 infectam os seres humanos (PANG J, et al., 2020).O sequenciamento do genoma e a análise filogênica indicaram que o coronavírus que causa a COVID-19 é um betacoronavírus com 75 a $80 \%$ de padrão de identificação com o vírus SARS-CoV (MCINTOSH K, et al., 2020; PANG J, et al., 2020; ZHOU P, et al., 2020).

Foi verificada também uma similaridade de sequência de RNA do SARS-CoV2 a de dois coronavírus de morcego, o que torna provável que eles sejam a fonte primária desta infecção (PERLMAN S, 2020; ZHOU $P$, et al., 2020). Ainda não se sabe se o vírus da COVID-19 é transmitido diretamente de morcegos ou através de algum outro mecanismo (por exemplo, um reservatório), o que se sabe é que quando um CoV animal, como o SARS-CoV-2, entra em um novo hospedeiro, como o ser humano, a gravidade da doença aumenta significativamente a cada nova rodada de adaptação (PANG J, et al., 2020; PERLMAN S, 2020).

Apesar de a causa exata da multiplicação viral precisar ser melhor entendida, acredita-se que o hospedeiro, e não apenas o vírus, desempenhe um papel fundamental na liberação de grandes quantidades de virions na multiplicação viral,_assim o resultado da infecção por SARS-CoV-2 é amplamente determinado pela interação vírus-hospedeiro (FUNG SY, et al., 2020; PANG J, et al., 2020). 
A comparação da sequência e organização do genoma de SARS-CoV e SARS-CoV-2 revela mais semelhanças do que diferenças. O SARS-CoV2 possui uma estrutura do domínio de ligação ao receptor muito similar ao coronavírus da SARS, demonstrado que o vírus usa o mesmo receptor. Assim o vibrião glicoproteína Spike (S) na superfície do coronavírus pode se ligar a enzima de conversão da angiotensina 2 (ECA2), fundindo-se à membrana das células e liberando o RNA viral (FUNG SY, et al., 2020; WU D, et al., 2020; ZHOU P, et al., 2020).

A infecção por SARS-CoV-2 desencadeada pela ligação da glicoproteína $S$ do vírus à a ECA2, afeta inúmeros órgão humanos pelo fato de a ECA2 está presente em abundância no coração (causando lesão miocárdica aguda pela infecção), nas células epiteliais do pulmão (resultando em sintomas respiratórios), intestino delgado, rins e vasos sanguíneos (FANG L, et al., 2020; PANG J, et al., 2020; RYU S, CHUN BC, 2020; ZHENG YY, et al., 2020).

Pacientes com doenças cardiovasculares tendem a apresentar sintomas mais graves em comparação a indivíduos saudáveis, uma vez que a expressão de ECA2 é substancialmente aumentada em pacientes com diabetes tipo 1 ou tipo 2 - tratados com inibidores da ECA e bloqueadores dos receptores da angiotensina (BRA) e a hipertensão - também tratada com inibidores da ECA e bloqueadores de receptores de angiotensina (BRA), o que pode estar associado ao aumento da secreção de ECA2 por regulação positiva. Consequentemente, o aumento da expressão de ECA2 facilitaria a infecção por COVID-19 (FANG L, et al., 2020; WAN Y, et al., 2020; ZHENG YY, et al., 2020).

Diante destas informações supõe-se que o tratamento do diabetes e da hipertensão com medicamentos estimulantes da ECA2 possa aumentar o risco de desenvolver COVID-19 grave e fatal (FANG L, et al., 2020). Em contra partida McIntosh K, et al. (2020) alertam que embora pacientes com doença cardiovascular, hipertensão e diabetes possam ter um curso clínico mais grave no cenário de infecção por SARS-CoV-2, não há evidências para apoiar uma associação com esses agentes.

Além disso, a interrupção desses agentes em alguns pacientes pode exacerbar doenças cardiovasculares ou renais comorbidades e levar ao aumento da mortalidade, não sendo, portanto, indicado a interrupção ou substituição do tratamento de pacientes que utilizam esses medicamentos (FANG L, et al., 2020).

\section{Transmissibilidade e período de incubação do vírus}

Ainda não é certo o tempo em que o indivíduo permanece transmitindo o COVID-19, apesar de alguns estudos mostrarem que o SARS-CoV2 pode ser transmitido antes da origem dos sintomas, também foi demonstrado que o período de maior infectividade é no estágio inicial da infecção. Portanto, muitos pacientes podem transmitir o vírus ainda no estágio de incubação (MCINTOSH K, et al., 2020). Segundo o estudo de Costa IBSS, et al. (2020) 85\% das transmissões ocorrem pelo contato direto com indivíduos assintomáticos.

Apesar da semelhança entre o SARS-Cov2 com os vírus que causaram as doenças em 2002 e 2012 , respectivamente Síndrome Respiratória do Oriente Médio (MERS) e SARS-CoV, o SARS-CoV2 tem transmissibilidade bem maior, sendo até 10 vezes mais transmissível que o SARS-CoV. No entanto, ao comparar o potencial de transmissão com o de outras doenças, a transmissibilidade é bem inferior quando comparado ao Sarampo.

Cada indivíduo infectado tem a capacidade de transmitir para em média três pessoas, o risco de transmissão de um indivíduo para outra varia conforme ao tipo de medidas preventivas utilizadas, ao tipo e a duração do contato e a fatores individuais de cada pessoa. (BRASIL, 2020a; COSTA IBSS, 2020; MCINTOSH $\mathrm{K}$, et al., 2020). Além da transmissão pessoa a pessoa por meio da transmissão de gotículas expelidas por meio da tosse e/ou espirro, o vírus também pode ser transmitido por meio de fômites, ou seja, através do contato com objetos manipulados pela pessoa infectada.

Ao analisar a presença do vírus em superfícies de ambientes onde pessoas com COVID-19 estavam internadas, foi detectado Ácido Ribonucleico (RNA) viral em diversos objetos, também foram colhidas amostras de objetos semelhantes de outros quartos de outros pacientes com COVID-19, mas nestes havia sido feita a limpeza usual que costuma ser feita no hospital, e não foi detectado RNA viral nesses locais.

REAS/EJCH | Vol.Esp.46 | e3762 | DOI: https://doi.org/10.25248/reas.e3762.2020 Página 4 de 8 
Mostrando que a limpeza se mostra eficaz na eliminação do vírus das superfícies e contribuindo para a diminuição da transmissão. O período de incubação é de 14 dias após exposição a fonte de contaminação, sendo que na maioria dos casos ocorre de 4 a 5 dias (MCINTOSH K, et al., 2020).

\section{Manifestações clínicas}

A ampla gama de apresentação clínica da COVID-19 pode variar de nenhum sintoma (assintomático) a casos graves de insuficiência respiratória com elevado potencial de óbito (HABIBZADEH P, STONEMAN EK, 2020; WU D, et al., 2020).

Os sinais e sintomas comuns mais prevalentes demonstrados em diferentes estudos foram: febre, tosse, dispneia, fadiga ou mialgia e de forma menos frequentes as apresentações intestinais (por exemplo a diarreia, náusea ou vômitos) (HABIBZADEH P, STONEMAN EK, 2020; LAI CC, et al., 2020; WU D, et al., 2020; YANG $\mathrm{J}$, et al., 2020). Outros sintomas menos citados, mas que também estão presente em muitos pacientes são: cefaleia persistente, desconforto na garganta, anosmia e ageusia, dando uma ênfase para a anosmia, visto que $80 \%$ dos pacientes que apresentavam esse sintoma tiveram o teste da RT-PCR do SARS-CoV2 positivo, sendo esse teste o padrão-ouro para o diagnóstico do COVID-19 (BRJUD MB, et al., 2020).

Pessoas afetadas pela COVID-19 que apresentam sinais e sintomas mais leves do tipo gripe, como os citados anteriormente, podem com a evolução da doença apresentar sintomas semelhantes a pneumonia, seguidos por uma infecção respiratória aguda grave com complicações que incluem desconforto respiratório agudo, lesão cardíaca aguda, arritmia, choque, falha de múltiplos órgãos, disfunção hepática, lesão renal aguda e infecção secundária (LAI CC, et al., 2020; MALIK YS, et al., 2020; WU D, et al., 2020).

As manifestações radiológicas mais comuns encontradas por tomografia computadorizada ou na radiografia de tórax também se tornaram úteis para avaliação das características clinico epidemiológicas de pacientes acometidos pela COVID-19, sendo a opacidade em vidro fosco a imagem de anormalidade mais comum, seguida de sombreamento irregular e anormalidades intersticiais. É importante ressaltar que o estágio da doença, a situação de imunidade do paciente, a idade do paciente, doenças subjacentes e a utilização de medicamentos podem interferir nos achados de imagens (LAI CC, et al., 2020; WU D, et al., 2020).

Segundo a investigação realizada por Wu D, et al. (2020), cerca de 60\% dos pacientes com COVID-19 e que apresentam pneumonia, também apresentam contagens normais ou diminuídas de glóbulos brancos e linfocitopenia. Em contra partida, a investigação realizada por Lai CC, et al. (2020) trouxe evidências que os casos de pneumonia apresentaram uma elevação de glóbulos brancos e de neutrófilos, mas com uma contagem reduzida de leucócitos quando comparados aos casos de Doença Respiratória Aguda (DRA). A divergência na literatura aponta para a necessidade de maiores esclarecimentos.

\section{Distribuição gênero e idade}

Embora a classificação por idade dos casos de COVID-19 nos diferentes estágios de gravidade esteja associada à criança, jovens e idosos, a maioria dos pacientes testados e diagnosticados com infecção pelo SARS-CoV-2 foi de 47 a 59 anos (HABIBZADEH P, STONEMAN EK, 2020; LAI CC, et al., 2020; YANG J, et al., 2020; YANG S, et al., 2020). Lai CC, et al. (2020) revelam em sua pesquisa que entre os casos de pneumonia, aproximadamente $15 \%$ das pessoas apresentavam idade superior a 65 anos.

A carga viral atinge o pico na primeira semana de infecção, ela se mostrou relacionada com a idade. A soroconversão ocorreu por volta da terceira semana. A alta carga viral durante o início pode ser o motivo da alta taxa de transmissibilidade e pode justificar o motivo da resistência aos antivirais. A carga viral se mostrou superior em pacientes idosos, mas não apenas pelo sistema imune baixo, também é relacionado com a quantidade de receptores ACE2 (receptor de entrada do vírus na célula) (CHEN Y, LI L, 2020).

Da distribuição por gênero, os pacientes do gênero masculinos compreenderam mais da metade dos casos da doença em todos os artigos analisados para esta pesquisa. (HABIBZADEH P, STONEMAN EK, 2020; LAI CC, et al., 2020; WU D, et al., 2020; YANG J, et al., 2020). Yang S, et al. (2020) ressaltam em seus achados que isso pode estar relacionado aos fatores de risco ocupacionais e pelo histórico de exposição de homens 
no mercado úmido de Huanan. Segundo Habibzadeh P e Stoneman EK (2020) os genes imunológicos relacionados ao cromossomo $\mathrm{X}$ e hormônios sexuais influenciam nas respostas imunes inatas e adaptativas relacionadas ao COVID 19. Entretanto, mais estudos são necessários para justificar esses achados e/ou confirmar essas hipóteses.

Estudo realizado por Zhou F, et al. (2020) com 191 pacientes, destes 137 receberam alta e 54 morreram. $48 \%$ desses pacientes tinham alguma comorbidade, dentre as mais observadas, podemos citar: diabetes, hipertensão e doença coronariana. A presença de comorbidades está relacionado com aumento dos níveis de mortalidade de quatro a cinco vezes e maior necessidade de internação em Unidade de Terapia Intensiva (UTI) (ESPER RN, et al., 2020; ZHOU F, et al., 2020)

\section{Infecções assintomáticas}

Embora os casos assintomáticos não apresentem sinais ou sintomas de infecção pelo SARS-CoV-2, esses merecem atenção das autoridades em saúde pelo fato de demonstrarem ser capazes de propagar o vírus de forma negligenciada a pessoas susceptíveis, desempenharem um papel critico no processo de transmissão da doença, tornando o controle da infecção um enorme desafio e tornando subestimado o número de pessoas infectadas pelo SARS-CoV-2 (FUNG SY, et al., 2020; LAI CC, et al., 2020; WU D, et al., 2020).

Estima-se que $85 \%$ das transmissões de COVID-19 tenham origem de pessoas assintomáticas, mesmo esses pacientes não apresentando sintomas ou sendo oligossintomáticos, não necessitam de hospitalização, mas tem papel fundamental para a disseminação da doença (COSTA IBSS, et al., 2020);

\section{Fatores de risco e COVID-19}

Ainda que os fatores de risco ou condições de suscetibilidade ligadas à patogênese da COVID-19 não estejam esclarecidos, os fatores do hospedeiro podem influenciar a suscetibilidade à infecção e à progressão da doença. Uma proporção significativa entre casos de infecção pelo SARS-CoV-2 e pacientes que apresentavam algum distúrbio coexistente como a hipertensão, o diabetes as doenças cardiovasculares, cerebrovasculares e doenças do aparelho respiratório foi relatada (YANG J, et al., 2020; LAI CC, et al., 2020; PANG J, et al., 2020; WU D, et al., 2020). Essa proporção pode, segundo Yang J, et al. (2020), estar relacionada ao fato de que doenças crônicas partilham características padrão como: distúrbios infecciosos, estado pró-inflamatório e a atenuação da resposta imune inata, que por sua vez interferem na resposta imune do paciente.

Além de estar associada a doenças de base, a susceptibilidade a infecção pelo SARS-CoV-2 também pode estar relacionada à idade dos pacientes, especialmente aqueles com hipertensão, doença cardíaca coronária ou diabetes. Assim idosos e aqueles com distúrbios subjacentes tendem a evoluir rapidamente para sintomas graves e condições críticas (LAI CC, et al., 2020; WU D, et al., 2020).

\section{Prognóstico}

Como tentativa de diminuir a rápida disseminação do vírus, a OMS recomendou o isolamento social, como tentativa de diminuir a superlotação de hospitais e garantir que os recursos necessários como leitos, ventilador mecânico e equipamentos de proteção individual para profissionais de saúde e pacientes. Essas medidas também são eficientes para fornecerem um tempo suficiente para os serviços de saúde se prepararem para receber e tratar esses pacientes (ANDERSON RM, et al, 2020; BRASIL, 2020b; JAWAID A, 2020). Na ausência de desenvolvimento de terapias e/ou vacinas para o tratamento do COVID-19, as medidas de isolamento social podem durar até 2022, não de maneira continua, mas passando por períodos intermitentes. Sendo essa forma de isolamento uma das medidas mais eficazes para evitar a sobrecarga dos serviços de saúde (KISSLER SM, et al., 2020).

A pandemia leva a impactos negativos sobre a economia mundial, abalando o comércio internacional e causando a possibilidade de uma crise financeira que pode levar há desequilíbrios severos na cadeia de produção, indo desde a quebra de um grande número de empresas, desemprego e crescimento acentuado da pobreza. Levando a impactos na economia mundial, visto que acabará afetando as importações e exportações mundiais, alterando a forma de fazer comércio entre os diversos países e causando 
desabastecimentos de alguns dos subsídios necessários para a sobrevivência da sociedade, gerando grande impacto na qualidade de vida e forte impacto na economia.

De certa forma, afetará os meios de subsistência, sendo diretamente prejudicados pela diminuição de suas fontes de renda, o que também trará consequências para sua saúde futura (ROUBICEK M, 2020; JONES L, BROW D e PALUMBO D, 2020; WANG YXJ, 2020).

\section{Perspectivas de tratamento}

O tratamento é pautado em medidas de suporte as possíveis disfunções orgânicas. Inicialmente foram testadas drogas antivirais, como a olsetamovir (utilizado para o tratamento da influenza), lopanovir e ritonavir (utilizados para o tratamento da Síndrome da Imunodeficiência Adquirida - AIDS) e remdesivir e interferon. Apesar de muitas delas demonstrarem eficácia moderada sobre a COVID-19, muitos deles são remédios de alto preço para a produção em larga escala e que não poderiam (COSTA IBSS, et al., 2020; AMIB, 2020).

Em estudo conduzido por Esper RN, et al. (2020) com uma população de 636 pacientes, destes, 64,7\% consentiram o tratamento com hidroxicloroquina e azitromicina e os demais não aceitaram o tratamento devido os riscos de efeitos colaterais ou não estavam possibilitados de tomar devido comorbidades que contraindicavam o uso. 1,9\% dos pacientes em uso dessas drogas necessitaram de internação, versus 5,4\% de internação do grupo que não estava em uso desses medicamentos. O estudo ainda comprovou que pacientes que iniciaram o tratamento com a associação de hidroxicloroquina e azitromicina antes do sétimo dia de sintomas tiveram uma evolução bem melhor do que os que iniciaram após o sétimo dia. Os tratamentos feitos com esses medicamentos ainda não possuem eficácia comprovada.

\section{CONSIDERAÇÕES FINAIS}

A recente infecção causada pelo SARS-CoV-2 traz consigo incertezas. os números são assustadores e a quantidade de mortos cresce vertiginosamente. Os estudos mostraram que o vírus tem um alto poder de transmissibilidade e por ainda não existir um tratamento específico para a doença, muitas pessoas estão sendo vítimas. Devido esse quadro de insegurança, faz-se necessário a disponibilizações de materiais que tragam para os profissionais da saúde atualizações sobre essa nova doença. Outro dado que assusta é a grande quantidade de casos assintomáticos, visto que essas pessoas transmitem o vírus da mesma forma que os sintomáticos. Atualmente existem muitas perspectivas de tratamento, mas ainda não existe estudos conclusivos que indiquem o melhor tratamento.

\section{REFERÊNCIAS}

1. ANDERSON RM, et al. How will country-based mitigation measures influence the course of the COVID-19 epidemic? Lancet. 2020;395(10228):931-934.

2. Associação de Medicina Intensiva Brasileira (AMIB). Terapia antimicrobiana empírica na síndrome respiratória aguda grave: pelo comitê de infecção e sepse da AMIB. 2020

3. BARJUD MB, et al. Protocolo para atendimento da COVID-19 no pronto atendimento. Médicos do Piauí. 2020.

4. BRASIL. Ministério da Saúde. Centro de Operações de emergências em Saúde Pública COE- nCoV. Boletim Epidemiológico 03. 2020a.

5. BRASIL. Ministério da Saúde. Centro de Operações de emergências em Saúde Pública COE- nCoV. Boletim Epidemiológico 08. 2020b

6. CHEN Y, LI L. SARS-CoV-2: virus dynamics and host response. The Lancet, 2020.

7. CUNNINGHAM AC, et al. Treatment of COVID-19: old tricks for new challenges. Crit Care. 2020; 24(1):91.

8. COSTA IBSS, et al. O Coração e a COVID-19: O que o Cardiologista Precisa Saber, Arq Bras Cardiol. 2020

9. ESPER RN, et al. Empirical treatment with hydroxychloroquine and azithromycin for suspected cases of COVID-19 followed-up by telemedicine. 2020.

10. FANG L, et al. Are patients with hypertension and diabetes mellitus at increased risk for COVID-19 infection? Lancet Respir Med. 2020; (20)30116-8.

11. FUNG SY, et al. A tug-of-war between severe acute respiratory syndrome coronavirus 2 and host antiviral defence: lessons from other pathogenic viruses. 2020; 9(1):558-570. 
12. GUO YR, et al. The origin, transmission and clinical therapies on coronavirus disease 2019 (COVID-19) outbreak - an update on the status. Mil Med Res. 2020; 7(1):11.

13. HABIBZADEH P, STONEMAN EK. The Novel Coronavirus: A Bird's Eye View. Int J Occup Environ Med. 2020; 11(2):65-71.

14. HOPKINS J. Mapa do coronavirus. Disponível em: https://coronavirus.jhu.edu/map.html.

15. JAWAID A. Protecting older adults during social distancing. Science. 2020;368(6487):145.

16. JONES L, BROWN D, PALUMBO D. Coronavírus: Oito gráficos que mostram o impacto da covid-19 sobre a economia mundial. BBC News Brasil. 2020. Disponível em: https://www.bbc.com/portuguese/internacional-51766452. Acesso em: 19 abr 2020.

17. KISSLER SM, et al. Projecting the transmission dynamic of SARS-CoV2 throught the postpandemic period. Science. 2020.

18. LAI CC, et al. Asymptomatic carrier state, acute respiratory disease, and pneumonia due to severe acute respiratory syndrome coronavirus 2 (SARS-CoV-2): Facts and myths. J Microbiol Immunol Infect. 2020.

19. MALIK YS, et al. Emerging novel coronavirus (2019-nCoV)-current scenario, evolutionary perspective based on genome analysis and recent developments. Vet Q. 2020; 40(1):68-76.

20. MCINTOSH K, et al. Coronavirus disease 2019 (COVID-19). Up ToDate. 2020.

21. NEHER RA, et al. Potential impact of seasonal forcing on a SARS-CoV-2 pandemic. Swiss Med Wkly. $2020 ; 156$.

22. PANG J, et al. Potential Rapid Diagnostics, Vaccine and Therapeutics for 2019 Novel Coronavirus (2019-nCoV): A Systematic Review. J Clin Med. 2020.

23. PERLMAN S. Another Decade, Another Coronavirus. N Engl J Med. 2020; 382(8):760-762.

24. ROUBICEK M. Qual o impacto da pandemia de coronavírus no comércio mundial. Nexo Jornal. 2020. Disponível em: https://www.nexojornal.com.br/expresso/2020/04/16/Qual-o-impacto-da-pandemia-de-coronav\%C3\%ADrus-nocom\%C3\%A9rcio-mundial. Acesso em: 19 abr 2020.

25. RYU S, CHUN BC. Korean Society of Epidemiology 2019-nCoV Task Force Team. An interim review of the epidemiological characteristics of 2019 novel coronavirus. Epidemiol Health. 2020; 42(e2020006).

26. VANKADARI N, WILCE JA. Emerging WuHan (COVID-19) coronavirus: glycan shield and structure prediction of spike glycoprotein and its interaction with human CD26. Emerg Microbes Infect. 2020; 9(1):601-604.

27. WAN Y, et al. Receptor recognition by novel coronavirus from Wuhan: An analysis based on decade-long structural studies of SARS. J Virology. 2020.

28. WANG YXJ, et al. The role of CT for Covid-19 patient's management remains poorly defined. Ann Transl Med. 2020;8(4):145.

29. WHO. World Health Organization. Coronavirus disease 2019 (COVID-19) Situation Report - 65. 2020.

30. WU D, et al. The SARS-CoV-2 outbreak: what we know. Int J Infect Dis. 2020; (20)30:123-5.

31. YANG J, et al. Prevalence of comorbidities in the novel Wuhan coronavirus (COVID-19) infection: a systematic review and meta-analysis. Int J Infect Dis. 2020; 12(20)30136-9.

32. YANG S, et al. Early estimation of the case fatality rate of COVID-19 in mainland China: a data-driven analysis. Ann Transl Med.2020; 8(4):128.

33. ZHENG YY, et al. COVID-19 and the cardiovascular system. Nat Rev Cardiol. 2020.

34. ZHOU P, et al. A pneumonia outbreak associated with a new coronavirus of probable bat origin. Nature. 2020; 579(7798):270-273.

35. ZHOU P, et al. Discovery of a novel coronavirus associated with the recent pneumonia outbreak in 2 humans and its potential bat origin. BioRxiv. 2020. 